\title{
Theoretical and Experimental Study of the Propagation and Divergence of Bounded Acoustic Beams
}

\author{
Jeroen Vandeputte and Oswald Leroy \\ Interdisciplinary Research Center, K.U. Leuven Campus Kortrijk, B-8500 Kortrijk, Belgium

\begin{abstract}
Abdellatif Bey Temsamani, Steve Vandenplas and Leo Van Biesen
Vrije Universiteit Brussel, Department of Fundamental Electricity and Instrumentation,

Pleinlaan 2, B-1050 Brussels, Belgium
\end{abstract}

(Received 3 May 2000; revised 24 October 2000; accepted 14 November 2000)

\begin{abstract}
In this paper, propagation and divergence effects of ultrasonic fields are studied. Four theoretical models are considered. Experiments were conducted in order to validate the results. The characteristics of the propagation and divergence of Gaussian acoustic beam are handled theoretically. It is shown that the propagation behaviour strongly depends on the material parameters and the initial halfwidth of the Gaussian beam. An experimentally measured profile at a distance $z$ from the emitter is used as boundary condition in the SDM-model and as a tool to estimate the parameters of the $\mathrm{CHW}$-model and the GBS-model by an inversion procedure. Comparison is made with experimental results.
\end{abstract}

\section{INTRODUCTION}

Real life measurements show that the distribution in an ultrasonic field is Gaussian. ${ }^{1}$ This makes Gaussian beams attractive as useful elements in field representation. As a result of recent analytical developments, any source field can be expressed exactly as a self-consistent superposition of Gaussian beams. This extends the use of Gaussian beams systematically to realistic transducer outputs. ${ }^{2}$ Because Gaussian beams have favourable propagation characteristics and represent physically observable entities, they have played a prominent role in many modelling schemes. ${ }^{3,4}$ However, scattering from localised fault zones or abrupt terminations are not beam - preserving. As a result the output for many transducers (such as a flat piston) gives rise to side lobes and other marked deviations from a well collimated Gaussian beam. For modelling the non-Gaussian effects without losing the characteristics of Gaussian beam propagation, the decomposition of the field into basic functions is required. ${ }^{5-8}$

In this paper, four theoretical approaches will be considered. All of them describe the spreading effects for a Gaussian distribution in an elastic isotropic liquid. Each of these models has its own characteristics for some specific cases. The aim of presenting these different approaches is the implementation of a toolbox of models, which can be useful to describe the major phenomena which occur during the ultrasonic beam propagation.

In the first section, the propagation and the spreading effects of one single Gaussian beam is explained in terms of the curvature of the wavefront and the changing halfwidth. In Section 3, the different theoretical approaches are studied. Decomposition into plane waves, the so-called Spectral Decomposition Model (SDM) and the Radiation Mode Model (RMM) are developed in the first two subsections. Two other models called the Gaussian Beam Superposition model
(GBS) and the Complex Harmonic Waves model (CHW) whose parameters are determined by the inversion of measured data, are described in Sections 3.3 and 3.4. In Section 4, comparisons with experimental results and discussion of the results are presented. Finally, conclusions are drawn.

\section{GENERAL CHARACTERISTICS OF A PROPAGATING BOUNDED BEAM}

We assume a transducer to be placed at $z=0$ which emits a plane Gaussian acoustic wave, which propagates in the $z$-direction (see Fig. 1). The displacement field at $z=0$ is given by (the factor $e^{-i \omega t}$ which depends on the angular frequency $\omega$, has been omitted):

$$
u(x, y, 0)=\sqrt{\frac{2}{\pi}} \frac{1}{W_{0}} e^{-\left(\frac{x^{2}+y^{2}}{W_{0}^{2}}\right)},
$$

where $W_{0}$ is the initial halfwidth.

After propagation over a distance $z$, the field can be written as: $:^{9,10}$

$$
u(x, y, z)=\sqrt{\frac{2}{\pi}} \frac{1}{W(z)} e^{-i(k z-\Psi(z))} e^{-i \frac{k}{2}\left(\frac{x^{2}+y^{2}}{R(z)}\right)} e^{-\left(\frac{x^{2}+y^{2}}{W^{2}(z)}\right)},
$$

where

$$
\begin{gathered}
R(z)=z+\left(\frac{\pi W_{0}^{2}}{\lambda}\right)^{2} \frac{1}{z} \\
W(z)=W_{0} \sqrt{1+\left(\frac{\lambda z}{\pi W_{0}^{2}}\right)^{2}} ;
\end{gathered}
$$

and

$$
\Psi(z)=\arctan \left(\frac{\lambda z}{\pi W_{0}^{2}}\right) .
$$

